\title{
Benign Pericardial Hemangioma-A Rare Cause of Cardiac Tamponade
}

\author{
Dhanya Jacob ${ }^{1}$ Thara Pratap ${ }^{1}$ Anand Kumar ${ }^{2} \quad$ Rashmi R. $^{3} \quad$ Vishnu A. K. ${ }^{1}$ \\ ${ }^{1}$ Department of Radiology, VPS Lakeshore Hospital, Kochi, Kerala, \\ India \\ 2 Department of Cardiology, VPS Lakeshore Hospital, Kochi, Kerala, \\ India \\ ${ }^{3}$ Department of Pathology, VPS Lakeshore Hospital, Kochi, Kerala, \\ India \\ Address for correspondence Vishnu A. K., MBBS, Rajeevam, House \\ No: 27, Swadeshabhimani Nagar, Neyyattinkara P.O., \\ Thiruvananthapuram 695 121, Kerala, India \\ (e-mail: drvishnuak@gmail.com). \\ Indian J Radiol Imaging 2021;31:754-757.
}

\begin{abstract}
Keywords

- cardiac tamponade

- computed tomography

- pericardial hemangioma

Pericardial tumors are very rare. It can be primary or secondary, of which secondary tumors are more common. Pericardial hemangiomas are extremely rare primary neoplasms and there are only very few cases published in the literature. These patients can be asymptomatic. When symptomatic, they present with dyspnea, palpitation, or atypical chest pain. Severity of symptoms depends on the size and location of the tumor. Pericardial effusion with features of cardiac tamponade can lead to a lifethreatening situation. Here, we report a case of pericardial hemangioma in a patient who presented with breathlessness and tamponade which was diagnosed preoperatively with computed tomography.
\end{abstract}

\section{Case Report}

A 66-year-old man presented with dyspnea on exertion of 6 months' duration. He was evaluated elsewhere with a chest radiograph and an echocardiogram. He was detected to have pericardial effusion and had undergone pericardiocentesis which was exudative in nature. Fluid cytology showed predominantly lymphocytes and was negative for malignant cells. Adenosine deaminase (ADA) was also negative. Though he had temporary relief of symptoms after aspiration, his symptoms worsened after a few days and was referred to our hospital with severe breathlessness. On admission, his blood pressure was $120 / 80 \mathrm{~mm} \mathrm{Hg}$ and pulse rate was $72 / \mathrm{min} \mathrm{He}$ was tachypnoeic. On examination, his heart sounds were muffled. Respiratory system examination showed normal vesicular breath sounds. Other systemic examinations were unremarkable. Chest radiograph showed marked enlargement of cardiac silhouette with cardiothoracic ratio of $94.4 \%$.
There were no features of pulmonary congestion (-Fig. 1). Provisional diagnosis of tuberculous/malignant pericardial effusion was considered. Tuberculosis was considered first because of the high prevalence in India. Malignancy was also considered as possibility because of the recurrent pericardial effusion. Complete hemogram was done. Hemoglobin was $14.6 \mathrm{~g} / \mathrm{dL}$. Total white blood cell count was $10.4 \times 103$, and erythrocyte sedimentation rate was $21 \mathrm{~mm} / \mathrm{h}$ (normal up to $10 \mathrm{~mm} / \mathrm{h}$ ). Echocardiogram done at our center showed presence of massive pericardial effusion ( - Fig. 2). No evidence of any debris, loculations, or septations was identified. An urgent pericardiocentesis was done and around $2,000 \mathrm{~mL}$ of straw-colored (nonhemorrhagic) fluid was aspirated. Fluid analysis showed predominant lymphocytes with no malignant cells, protein $-4.0 \mathrm{~g} / \mathrm{dL}$, and sugar $-132 \mathrm{mg} / \mathrm{dL}$. TB gold, acid-fast bacillus (AFB) stain, and AFB culture of fluid were negative. Pericardial fluid ADA (ADA level) was normal $(6 \mathrm{U} / \mathrm{L})$.
DOI https://doi.org/ 10.1055/s-0041-1736405. ISSN 0971-3026.

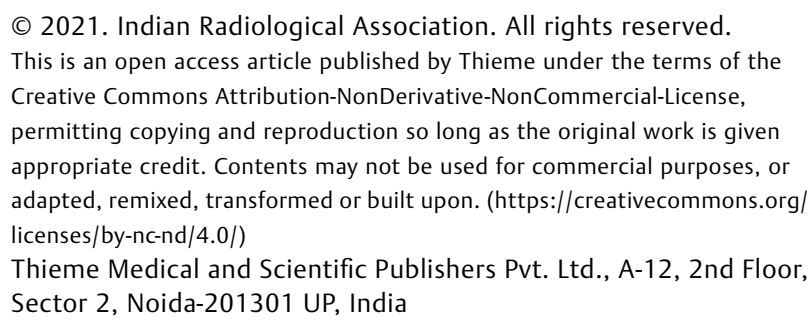




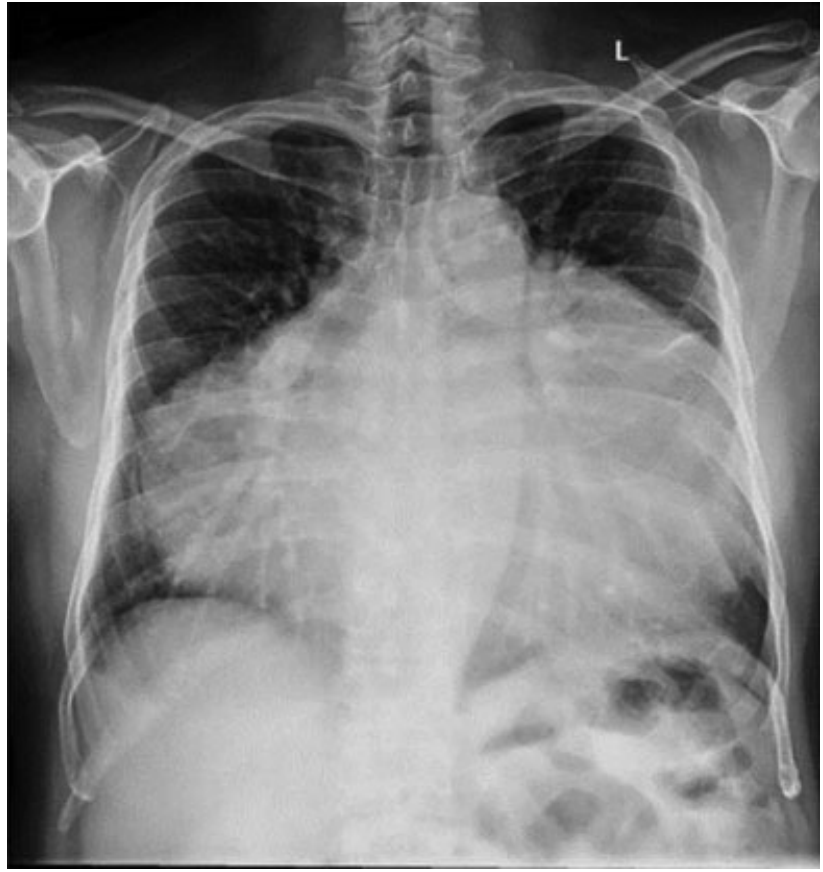

Fig. 1 Frontal radiograph showing markedly enlarged cardiac silhouette with sharp margins indicating massive pericardial effusion.

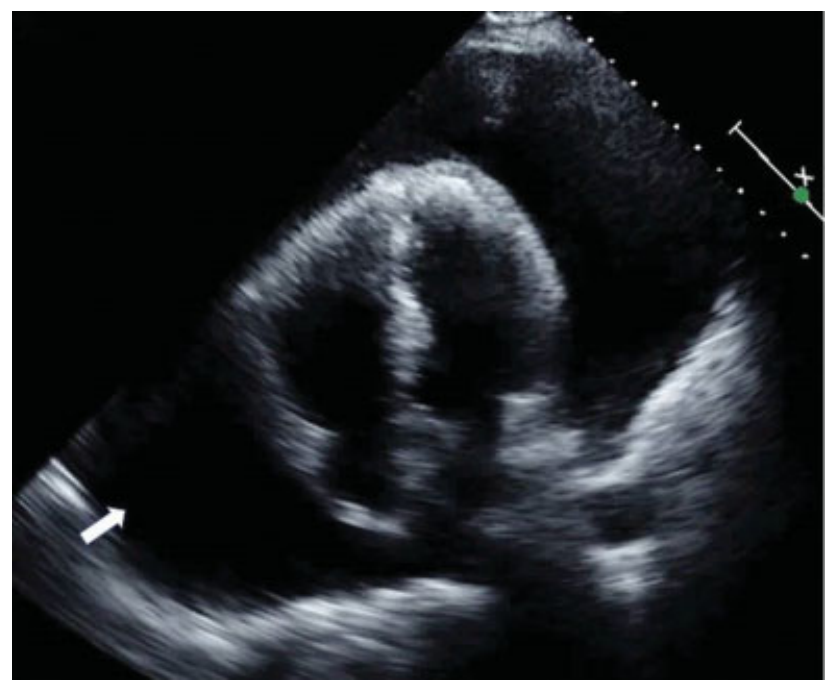

Fig. 2 Two-dimensional echocardiography showing significant pericardial effusion (white arrow).

Tumor markers were done to exclude malignant etiology. Carcinoembryonic antigen $(17.4 \mathrm{ng} / \mathrm{mL}$, normal $<3 \mathrm{mg} / \mathrm{mL})$, prostate-specific antigen, cancer antigen 19-9, and alphafetoprotein were normal. He was symptomatically better and got discharged. Again, he presented with the same symptoms after 2 weeks. He again underwent urgent pericardiocentesis and $1,900 \mathrm{~mL}$ of straw-colored fluid was aspirated.

Computed tomography (CT) scan of the thorax and abdomen, and colonoscopy were done to look for primary malignancy in view of recurrent pericardial effusion.

Electrocardiogram-gated cardiac multidetector CT scan of the thorax revealed a soft tissue mass lesion with lobulated margins measuring $3.6 \times 3.6 \mathrm{~cm}$ within the pericardium abutting the main and left pulmonary arteries. Left anterior

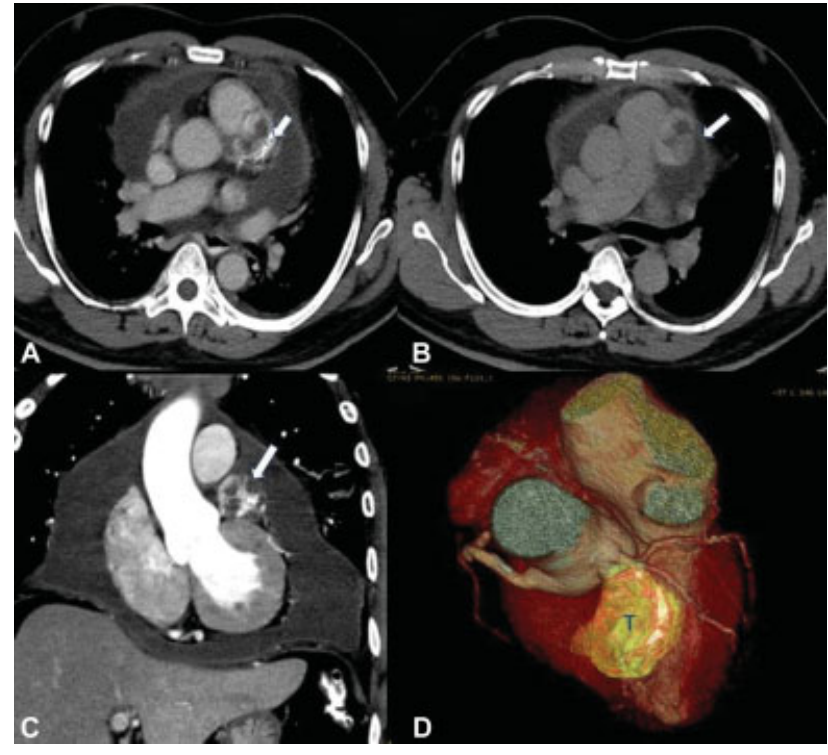

Fig. $3 \mathrm{MDCT}(\mathrm{A}-\mathrm{D})$ showing heterogeneous appearing pericardial hemangioma (white arrow in $\mathrm{A}-\mathrm{C}$ ) with peripheral enhancement in early phase and progressive enhancement in late phase (axial sections A, B). Coronal images (C) and volume rendered images (D) show the lesion ( $\mathrm{T}$ ) below the MPA abutting LAD. LAD, left anterior descending artery; MDCT, multidetector computed tomography; MPA, main pulmonary artery; $\mathrm{T}$, tumor.

descending artery was seen stretched posterior to the mass. There was no calcification. The lesion showed intense peripheral enhancement with gradual filling in and homogenous enhancement in delayed phase (-Fig. 3A-D).

In view of recurrent symptoms, surgical resection of lesion was planned, limited thoracotomy showed an encapsulated mass within the pericardium abutting main pulmonary artery, anterior to left anterior descending artery (-Fig. 4). The mass was removed and was sent for histopathological examination.

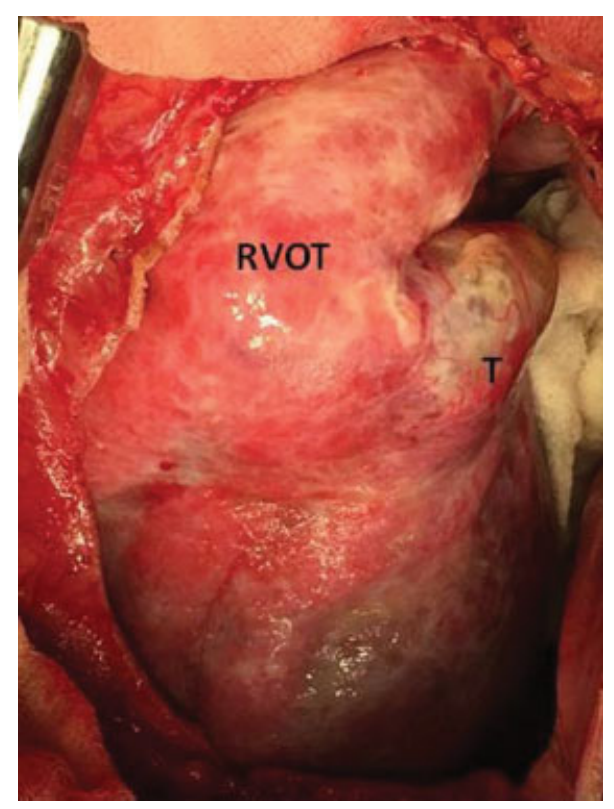

Fig. 4 Intraoperative picture showing pericardial lesion close to the main pulmonary artery. RVOT, right ventricle outflow tract; $\mathrm{T}$, tumor. 


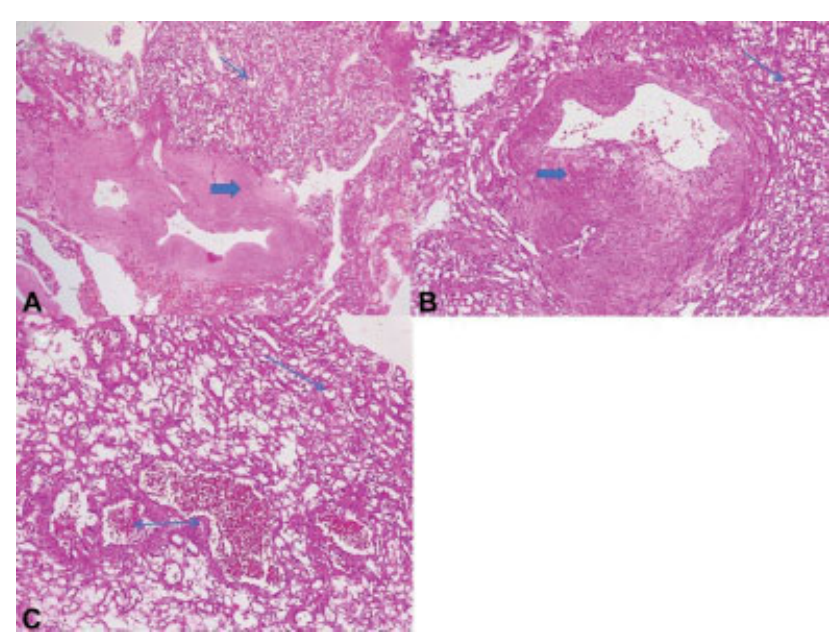

Fig. 5 (A) Scanner view of the intrapericardial mass shows a neoplasm composed of variably sized blood vessels. The tumor is predominantly composed of small-sized thin-walled vessels of capillary type hemangioma (thin arrow) along with large irregular thick-walled vessels of arteriovenous type hemangioma (thick arrow). (B) Low power view shows vessels with arteriovenous type hemangioma (thick arrow) and capillary type hemangioma (thin arrow). (C) Low power view showing capillary type hemangioma (single arrow) with few cavernous type vascular channels (doubleheaded arrow).

The gross specimen measured $4 \times 3 \times 3 \mathrm{~cm}$ with brownish cut section. Histopathology showed a vascular neoplasm composed predominantly of capillary type hemangioma having capillary-sized small thin-walled vascular channels with feeder vessels. Focal areas with dilated cavernous blood-filled, thin-walled vascular spaces of cavernous type hemangioma and malformed thick- and thin-walled vascular channels of arteriovenous type of hemangioma were also seen. The vessels were lined by bland endothelial cells (-Fig. 5A-C).

\section{Discussion}

Primary cardiac tumors are exceedingly rare tumors. ${ }^{1}$ Tumors primarily arising from pericardium are even rarer. According to a case series studied by Meng et al and Patel and Sheppard, ${ }^{2,3}$ the prevalence of primary pericardial neoplasms is around 0.001 to $0.007 \%$. Of the primary pericardial masses, pericardial cyst is the most common. Other common benign neoplasms are lipoma, lipoblastoma, paraganglioma, germ cell tumor, pericardial hemangioma, fibroma, and inflammatory pseudotumor. Mesothelioma is the most common primary pericardial malignancy. ${ }^{4}$

Cardiac hemangiomas comprise only 2 to $5 \%$ of benign cardiac tumors. It can occur at any age and can be single or rarely multiple. Rarely, it can be associated with hemangiomas at extrathoracic sites such as gastrointestinal tract, skin, or face. ${ }^{5} \mathrm{CT}$ of the thorax and abdomen in our case did not reveal any other hemangiomas. Pericardial hemangiomas are extremely rare and most are found incidentally during autopsy. ${ }^{6}$ Most of the patients are asymptomatic. In symptomatic patients, the severity of symptoms depends on the size and location of the tumor. The common symptoms are dysrhythmia, breathlessness, chest pain, cough, or dysphagia. It can produce pericardial effusion which may be serous or hemorrhagic, the mechanism of which is not well understood. The possible explanation for serous effusion is that it could be due to pericardial friction. Hemorrhagic effusion can occur due to rupture of tumorous microvessels. ${ }^{7}$ Sometimes pericardial hemangiomas can cause tamponade and sudden death. ${ }^{8}$ In the case series of 12 pericardial hemangiomas studied by Seitz et al, only three patients presented with pericardial effusion. Two cases were detected as an incidental finding. ${ }^{7}$ Our case was symptomatic with pericardial effusion and features of cardiac tamponade.

Diagnostic modalities for the evaluation of cardiac tumors are transthoracic echocardiography, $\mathrm{CT}$, magnetic resonance imaging (MRI), or cardiac catheterization. Transesophageal echocardiography is more useful than transthoracic echocardiography to detect cardiac and pericardial tumors. Echocardiography is a sensitive noninvasive tool in suspected intracardiac tumors as a preliminary investigation and can be used to exclude other tumors such as left atrial myxoma. Though sensitivity of echocardiography in diagnosing a pericardial tumor is limited, flow signals in Doppler echocardiography may give a clue toward hemangioma. However, in our case, the tumor was not identified on echocardiography. This may be attributed to the massive pericardial effusion which may have rendered the visualization of the tumor difficult. Also, the procedure was done on an emergency setting with the primary goal of relieving the patient distress by pericardiocentesis. Other factors that may have contributed to the nonvisualization of the tumor may include the small size of the tumor and its anterior location behind the sternum. A relook echo was done after the CT which showed a suspicious focal lesion adjacent to the right ventricular outflow tract.

A preoperative diagnosis was possible with $\mathrm{CT}$ scan which showed typical features of a vascular tumor arising from the pericardium. Because of the vascularity, hemangiomas show intense contrast enhancement in early arterial phase and this typical feature was demonstrable in our case. However, in some previous reports of similar cases, it was difficult to differentiate the benign pericardial tumors preoperatively without histology in spite of multiple imaging modalities. ${ }^{9,10}$ We feel that a multidetector $\mathrm{CT}$ is a good imaging modality for larger pericardial hemangiomas presenting with significant pericardial effusion.

MRI is a very good modality and can be performed as a problem solving tool. Due to excellent contrast resolution and multiplanar capability, MRI allows optimal evaluation of myocardial infiltration, pericardial involvement, and extracardiac extension of cardiac tumors. At MRI, the hemangioma shows intermediate T1-weighted signal intensity and high T2-weighted signal intensity and nodular areas of intense enhancement in early postcontrast images with progressive filling on delayed images. ${ }^{4}$ Coronary angiography is another useful modality which can demonstrate the feeder vessel to the tumor with characteristic tumor blush. ${ }^{11}$ Tumor blush is commonly seen in the capillary and 
arteriovenous types of hemangiomas due to rapid blood flow. ${ }^{12}$

Cardiac hemangiomas arise from the endocardium, myocardium, or epicardium/pericardium. They can occur at any chamber and most commonly found to arise from the ventricles followed by atria. ${ }^{1}$ Histologically, it is classified into capillary (composed of smaller capillary-like vessels), cavernous (composed of multiple thin-walled, dilated vessels), and arteriovenous (composed of thick-walled dysplastic arteries, venous-like vessels, and capillaries). ${ }^{13}$ Pericardial hemangiomas mostly arise from visceral pericardium and are usually cavernous type. ${ }^{4}$ In our case, the tumor was arising from visceral pericardium abutting the pulmonary artery. Among the pericardial hemangiomas presented with pericardial effusion in the case series of Seitz et al, the tumor was seen arising from anterior wall of left ventricle in one case and in the second case, it was originating from pericardium next to pulmonary artery as in our case. ${ }^{7,14}$ In another similar case, the tumor was located in between left atrial appendage and pulmonary vein. ${ }^{11}$ All these three cases proved to be capillary type hemangioma on histology. Our case is unique with histology showing predominantly capillary type with admixtures of cavernous and arteriovenous components.

Surgical excision is the treatment of choice for cardiac hemangioma. The prognosis of patients with surgically treated symptomatic lesions is excellent. ${ }^{15}$ If untreated, it can cause complications such as cardiac tamponade and sudden death. Spontaneous regression of the tumor has also been reported. ${ }^{16}$ There are reports of tumor regression after steroid treatment and also with radiotherapy. ${ }^{17}$ Our patient who presented with life-threatening cardiac tamponade was successfully managed with surgical excision. Recurrence is possible especially if incompletely removed. This patient was followed up for 4 years and no recurrence was noted. This in turn shows that a complete removal was achieved during surgery.

\section{Conclusion}

Pericardial hemangiomas are extremely rare. Our case presented with recurrent episodes of pericardial effusion and features of tamponade. A proper preoperative diagnosis was possible as multidetector CT showed typical features of vascular pericardial lesion. A complete removal of the tumor was attained surgically. We report this case as a unique one due to the extreme rarity of pericardial location of hemangiomas and also the presence of all three histologic types in the same tumor.

\section{Declaration of Patient Consent}

The authors certify that they have obtained all appropriate patient consent forms. In the form, the patient(s) has/have given his/her/their consent for his/her/their images and other clinical information to be reported in the journal. The patients understand that their names and initials will not be published and due efforts will be made to conceal their identity, but anonymity cannot be guaranteed.

\section{Financial Support and Sponsorship}

Nil.

\section{Conflict of Interest}

None declared.

\section{References}

1 Vargis RS, Phansalkar M, Padhi S, Phansalkar D, Nair SR. Pericardial hemangioma: a common tumour in an unusual location: Case report and review of literature. J Clin Diagn Res 2017;11(05): ED15-ED17

2 Meng Q, Lai H, Lima J, Tong W, Qian Y, Lai S. Echocardiographic and pathologic characteristics of primary cardiac tumors: a study of 149 cases. Int J Cardiol 2002;84(01):69-75

3 Patel J, Sheppard MN. Pathological study of primary cardiac and pericardial tumours in a specialist UK Centre: surgical and autopsy series. Cardiovasc Pathol 2010;19(06):343-352

4 Restrepo CS, Vargas D, Ocazionez D, Martínez-Jiménez S, Betancourt Cuellar SL, Gutierrez FR. Primary pericardial tumors. Radiographics 2013;33(06):1613-1630

5 Lamba G, Frishman WH. Cardiac and pericardial tumors. Cardiol Rev 2012;20(05):237-252

6 Akikwala T, Trivedi D, Kochamba G, et al. Perioperative evaluation and surgical management of a patient with a pericardial hemangioma abutting the right ventricular outflow tract and main pulmonary artery. J Cardiothorac Vasc Anesth 2019;33(05): 1362-1366

7 Seitz A, Ong P, Backes M, Mahrholdt H. Chronic pericardial effusion in the setting of pericardial capillary haemangioma: a case report and review of the literature. Eur Heart J Case Rep 2018;2(01):yty024

8 Sabeti S, Zahedifard S, Soleimantabar H, Zarghampour M, Toutkaboni M. Coexistence of pericardialand hepatic hemangiomas. Iran J Pathol 2015;10(02):169-172

9 Abuharb MYI, Bian XM, He J. Epicardial cardiac cavernous haemangioma-a case report. BMC Cardiovasc Disord 2019;19(01): 179

10 O'Brien EM, Low YH. Epicardial cavernous hemangioma: a diagnostic challenge. CASE (Phila) 2018;2(06):262-265

11 Omura K, Onishi T, Yamawaki K, et al. A rare case of pericardial hemangioma with bloody pericardial effusion. J Cardiol Cases 2010;2(01):e15-e19

12 Tadros NB, Akl BF, Avasthi P, et al. Arteriovenous and capillary hemangiomas of the interventricular septum. Ann Thorac Surg 1988;46(02):236-238

13 Grebenc ML, Rosado de Christenson ML, Burke AP, Green CE, Galvin JR. Primary cardiac and pericardial neoplasms: radiologicpathologic correlation. Radiographics 2000;20(04):1073-1103, quiz 1110-1111, 1112

14 Liebetrau C, Szalay Z, von Gerlach S, et al. Pericardial hemangioma vascularized via left anterior descending. Clin Res Cardiol 2010;99 (06):405-407

15 Gupta N. Intrapericardial hemangioma: a case report. J Clin Diagn Res 2013;7(01):169-170

16 Brizard C, Latremouille C, Jebara VA, et al. Cardiac hemangiomas. Ann Thorac Surg 1993;56(02):390-394

17 Wu G, Jones J, Sequeira IB, Pepelassis D. Congenital pericardial hemangioma responding to high-dose corticosteroid therapy. Can J Cardiol 2009;25(04):e139-e140 